\title{
ERRATUM: Investigation of Feline calicivirus infection in cats with upper respiratory tract disease in Diyarbakir, Turkey
}

\author{
Nazan Baksi ${ }^{1}$ (D); Aynur Simsek ${ }^{1}$
}

${ }^{1}$ Dicle University, Veterinary Faculty, Department of Internal Medicine, Diyarbakir, Turkey

In the article "Investigation of Feline calicivirus infection in cats with upper respiratory tract disease in Diyarbakir, Turkey” (DOI https://doi.org/10.11606/issn.1678-4456.bjvras.2021.177172), published in Braz J Vet Res Anim Sci. 2021;58:e177172.

On page 4, where the Table 2 shows:

Table 2 - Feline calicivirus investigation. Clinical examination findings in cats according to the group. Diyarbakir region, Turkey, between March 2018 and January 2019.

\begin{tabular}{cccc}
\hline Parameters & Control group $(\mathbf{n}=\mathbf{1 0})$ & Cats with URTD $(\mathbf{n}=\mathbf{2 0})$ & P value $^{*}$ \\
\hline $\mathrm{T}\left({ }^{\circ} \mathrm{C}\right)$ & $38,17 \pm 0,10^{* *}$ & $38,32 \pm 0,18$ & 0,18 \\
$\mathrm{R}(/ \mathrm{min})$ & $43,80 \pm 2,32$ & $28,65 \pm 1,53$ & 0,00 \\
$\mathrm{HR}(/ \mathrm{min})$ & $137,4 \pm 6,15$ & $122,95 \pm 4,38$ & 0,07 \\
\hline
\end{tabular}

T: Temperature; R: respiration rate; HR: heart rate; min: minute. *: $\mathrm{p}<0.05 ;$ ** (mean $\pm \mathrm{SEM})$.

It should show:

Table 2. Feline calicivirus investigation. Clinical examination findings (mean \pm SEM) in cats according to the group. Diyarbakir region, Turkey, between March 2018 and January 2019.

\begin{tabular}{cccc}
\hline Parameters & Control group $(\mathbf{n}=\mathbf{1 0})$ & Cats with URTD $(\mathbf{n}=\mathbf{2 0})$ & P value \\
\hline $\mathrm{T}\left({ }^{\circ} \mathrm{C}\right)$ & $38,17 \pm 0,10$ & $38,32 \pm 0,18$ & 0,18 \\
$\mathrm{R}(/ \mathrm{min})$ & $43,80 \pm 2,32$ & $28,65 \pm 1,53$ & $0,00^{*}$ \\
$\mathrm{HR}(/ \mathrm{min})$ & $137,4 \pm 6,15$ & $122,95 \pm 4,38$ & 0,07 \\
\hline
\end{tabular}

T: Temparature; R: respiration rate; HR: heart rate; min: minute; *: p $<0.05$

On page 4, where the Table 3 shows:

Table 3 - Feline calicivirus investigation. Hematological findings of cats, according to the group. Diyarbakir region, Turkey, between March 2018 and January 2019

\begin{tabular}{|c|c|c|c|}
\hline Parameters & Control group $(n=10)$ & Cats with URTD $(n=20)$ & P value* \\
\hline WBC $\left(\mathrm{m} / \mathrm{mm}^{3}\right)$ & $18,85 \pm 3,68^{* *}$ & $25,44 \pm 3,50$ & 0,25 \\
\hline $\operatorname{Lym}\left(\mathrm{m} / \mathrm{mm}^{3}\right)$ & $5,58 \pm 1,01$ & $7,07 \pm 1,36$ & 0,48 \\
\hline $\operatorname{Mon}\left(\mathrm{m} / \mathrm{mm}^{3}\right)$ & $0,71 \pm 0,13$ & $1,26 \pm 0,74$ & 0,25 \\
\hline $\operatorname{Gra}\left(\mathrm{m} / \mathrm{mm}^{3}\right)$ & $12,56 \pm 3,11$ & $17,09 \pm 2,58$ & 0,30 \\
\hline Lym (\%) & $32,22 \pm 4,72$ & $27,49 \pm 2,91$ & 0,38 \\
\hline Mon (\%) & $4,03 \pm 0,11$ & $5,30 \pm 0,42$ & 0,04 \\
\hline Gra (\%) & $63,75 \pm 4,77$ & $66,88 \pm 3,05$ & 0,57 \\
\hline $\mathrm{RBC}\left(\mathrm{m} / \mathrm{mm}^{3}\right)$ & $8,94 \pm 0,97$ & $9,76 \pm 0,69$ & 0,50 \\
\hline HGB (g/dL) & $14,30 \pm 1,33$ & $14,38 \pm 1,07$ & 0,96 \\
\hline HCT (\%) & $43,25 \pm 3,91$ & $45,14 \pm 3,15$ & 0,72 \\
\hline $\mathrm{MCV}(\mathrm{g} / \mathrm{dL})$ & $49,24 \pm 1,39$ & $46,55 \pm 1,11$ & 0,16 \\
\hline $\mathrm{MCH}(\mathrm{g} / \mathrm{dL}$ & $16,16 \pm 0,39$ & $14,63 \pm 0,34$ & $0,01^{*}$ \\
\hline $\mathrm{MCHC}(\mathrm{g} / \mathrm{dL})$ & $32,93 \pm 0,23$ & $31,66 \pm 0,30$ & $0,01^{*}$ \\
\hline Plt $\left(\mathrm{m} / \mathrm{mm}^{3}\right)$ & $199,2 \pm 39,42$ & $109,9 \pm 16,20$ & $0,02 *$ \\
\hline
\end{tabular}

WBC: White blood cell; Lymph: Lymphocyte; Mon: Monocyte; Gran: granulocyte; RBC: Red blood cell; HGB: Hemoglobin; HCT: Hematocrit; MCV: Mean corpuscular volume; MCH: Mean corpuscular hemoglobin in erythrocytes; MCHC: Mean corpuscular hemoglobin concentration; PLT: Platelet.

It should show: 
Table 3. Feline calicivirus investigation. Hematological findings (mean \pm SEM) of cats, according to the group. Diyarbakir region, Turkey, between March 2018 and January 2019

\begin{tabular}{|c|c|c|c|}
\hline Parameters & Control group $(n=10)$ & Cats with URTD $(n=20)$ & P value \\
\hline WBC $\left(\mathrm{m} / \mathrm{mm}^{3}\right)$ & $18,85 \pm 3,68$ & $25,44 \pm 3,50$ & 0,25 \\
\hline $\operatorname{Lym}\left(\mathrm{m} / \mathrm{mm}^{3}\right)$ & $5,58 \pm 1,01$ & $7,07 \pm 1,36$ & 0,48 \\
\hline $\operatorname{Mon}\left(\mathrm{m} / \mathrm{mm}^{3}\right)$ & $0,71 \pm 0,13$ & $1,26 \pm 0,74$ & 0,25 \\
\hline $\operatorname{Gra}\left(\mathrm{m} / \mathrm{mm}^{3}\right)$ & $12,56 \pm 3,11$ & $17,09 \pm 2,58$ & 0,30 \\
\hline Lym (\%) & $32,22 \pm 4,72$ & $27,49 \pm 2,91$ & 0,38 \\
\hline Mon (\%) & $4,03 \pm 0,11$ & $5,30 \pm 0,42$ & $0,04^{*}$ \\
\hline Gra (\%) & $63,75 \pm 4,77$ & $66,88 \pm 3,05$ & 0,57 \\
\hline $\mathrm{RBC}\left(\mathrm{m} / \mathrm{mm}^{3}\right)$ & $8,94 \pm 0,97$ & $9,76 \pm 0,69$ & 0,50 \\
\hline HGB (g/dL) & $14,30 \pm 1,33$ & $14,38 \pm 1,07$ & 0,96 \\
\hline НCT (\%) & $43,25 \pm 3,91$ & $45,14 \pm 3,15$ & 0,72 \\
\hline $\mathrm{MCV}(\mathrm{g} / \mathrm{dL})$ & $49,24 \pm 1,39$ & $46,55 \pm 1,11$ & 0,16 \\
\hline $\mathrm{MCH}(\mathrm{g} / \mathrm{dL}$ & $16,16 \pm 0,39$ & $14,63 \pm 0,34$ & $0,01 *$ \\
\hline $\mathrm{MCHC}(\mathrm{g} / \mathrm{dL})$ & $32,93 \pm 0,23$ & $31,66 \pm 0,30$ & $0,01 *$ \\
\hline Plt $\left(\mathrm{m} / \mathrm{mm}^{3}\right)$ & $199,2 \pm 39,42$ & $109,9 \pm 16,20$ & $0,02^{*}$ \\
\hline
\end{tabular}

WBC: Total leukocyte count; Lymph: Lymphocyte; Mon: Monocyte; Gran: granulocyte; RBC: Red blood cell; HGB: Hemoglobin; HCT: Hematocrit; MCV: Mean corpuscular volume; MCH: mean corpuscular hemoglobin in erythrocytes; MCHC: Mean corpuscular hemoglobin concentration; PLT: Platelet.; *: p < 0.05;

On page 4, where the Table 4 shows:

Table 4 - Feline calicivirus investigation. Serum biochemistry findings of cats, according to the group. Diyarbakir region, Turkey, between March 2018 and January 2019

\begin{tabular}{cccc}
\hline Parameters & Control group $(\mathbf{n = 1 0})$ & Cats with URTD $(\mathbf{n}=\mathbf{2 0})$ & P value $^{*}$ \\
\hline BUN $(\mathrm{mg} / \mathrm{dL})$ & $22,86 \pm 1,36^{* *}$ & $22,66 \pm 1,27$ & 0,92 \\
Cre $(\mathrm{mg} / \mathrm{dL})$ & $1,24 \pm 0,14$ & $0,88 \pm 0,08$ & 0,02 \\
ALT (U/L) & $67,60 \pm 15,75$ & $85,30 \pm 11,42$ & 0,37 \\
\hline
\end{tabular}

BUN: Blood urea nitrogen; Cre: Creatinine; ALT: Alanine aminotransferase *: $\mathrm{p}<0.05$.

It should show:

Table 4. Feline calicivirus investigation. Serum biochemistry findings (mean \pm SEM) of cats, according to the group. Diyarbakir region, Turkey, between March 2018 and January 2019

\begin{tabular}{cccc}
\hline Parameters & Control group $(\mathbf{n}=\mathbf{1 0})$ & Infected group $(\mathbf{n}=\mathbf{2 0})$ & P value \\
\hline BUN $(\mathrm{mg} / \mathrm{dL})$ & $22,86 \pm 1,36$ & $22,66 \pm 1,27$ & 0,92 \\
Cre $(\mathrm{mg} / \mathrm{dL})$ & $1,24 \pm 0,14$ & $0,88 \pm 0,08$ & $0,02^{*}$ \\
ALT $(\mathrm{U} / \mathrm{L})$ & $67,60 \pm 15,75$ & $85,30 \pm 11,42$ & 0,37 \\
\hline
\end{tabular}

BUN: Blood urea nitrogen; Cre: Creatinine; ALT: Alanine aminotransferase; *: $<<0.05$ 